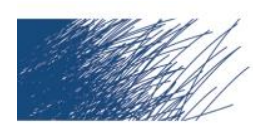

\title{
$\underline{\underline{\text { IIIII }}}$ \\ UNIVERSITEIT GENT
}

biblio.ugent.be

The UGent Institutional Repository is the electronic archiving and dissemination platform for all UGent research publications. Ghent University has implemented a mandate stipulating that all academic publications of UGent researchers should be deposited and archived in this repository. Except for items where current copyright restrictions apply, these papers are available in Open Access.

This item is the archived peer-reviewed author-version of:

Title: Are capillary dried blood spots applicable for therapeutic drug monitoring of common antipsychotics? A proof of concept

Authors: Patteet L., Maudens K., Stove C., Lambert W., Morrens M., Sabbe B., Neels H. In: Bioanalysis, 7 (16) 2119-2130, 2015.

To refer to or to cite this work, please use the citation to the published version:

Patteet L., Maudens K., Stove C., Lambert W., Morrens M., Sabbe B., Neels H. (2015). Are capillary dried blood spots applicable for therapeutic drug monitoring of common antipsychotics? A proof of concept. Bioanalysis 7 (16) 2119-2130. DOI 10.4155/bio.15.100 
Are capillary dried blood spots applicable for therapeutic drug monitoring of common antipsychotics? A proof of concept

Lisbeth Patteet ${ }^{1,2}$; Kristof E. Maudens $(\mathrm{PhD})^{1}$; Christophe P. Stove $(\mathrm{PhD})^{3}$; Willy E. Lambert

$(\mathrm{PhD})^{3}$; Manuel Morrens (MD, PhD $)^{4,5}$; Bernard Sabbe $(\mathrm{MD}, \mathrm{PhD})^{4,6}$; Hugo Neels $(\mathrm{PhD})^{1,2}$

1 Toxicological Centre University of Antwerp

Universiteitsplein 1

B-2610 Antwerp, Belgium

2 Laboratory for TDM and Toxicology

ZNA Stuivenberg

Lange Beeldekensstraat 267

B-2060 Antwerp, Belgium

3 Laboratory of Toxicology

Ghent University

Ottergemsesteenweg 460

B-9000 Ghent, Belgium

4 Collaborative Antwerp Psychiatric Research Institute (CAPRI)

Faculty of Medicine

University of Antwerp

Universiteitsplein 1

B-2610 Antwerp, Belgium

5 Psychiatric Hospital Broeders Alexianen

Provinciesteenweg 408

B-2530 Boechout, Belgium

6 Psychiatric Centre Sint-Norbertushuis

Stationstraat $22 \mathrm{C}$

B-2570 Duffel, Belgium

Corresponding author: Lisbeth Patteet

Postal address: Toxicological Centre, University of Antwerp, Universiteitsplein 1, B-2610

Wilrijk, Belgium.

Telephone: 32-3-2652704

Fax: 32-3-2652722

E-mail : lisbeth.patteet@uantwerpen.be 


\title{
Key Terms
}

Dried blood spot sampling: A micro-sampling technique where a small volume $(<100 \mu \mathrm{L})$ of blood obtained by finger prick is spotted on special filter paper

Therapeutic drug monitoring: The measurement of a parameter (usually a xenobiotic) that, with appropriate interpretation, can directly influence prescription of a medication.

Blood: serum ratio: Ratio of the drug concentration in blood to the concentration in serum. A critical parameter for calculating the corresponding serum concentration from dried blood spot results.

Therapeutic range: A population-based range of drug concentrations with a defined lower limit, below which a drug induced therapeutic response is less expected, and an upper limit, above which there is a higher chance for toxicity and serious adverse effects without further improvement of the therapeutic response.

Long-acting injectable formulation: Long-acting or depot formulations of drugs are intramuscular formulations which gradually release the active compound in the systemic circulation over a longer period of time, usually 2-4 weeks. In psychiatry, they are used to overcome the non-adherence problem observed in $40-60 \%$ of patients.

\begin{abstract}
Abbreviations
Capillary dried blood spot, c-DBS; Daily dose, DD; Dried blood spot, DBS; Dynamic multiple reaction monitoring, dMRM; Electrochemical detection, ECD; International Association of Therapeutic Drug Monitoring and Clinical Toxicology, IATDMCT; Liquid chromatography, LC; Lower limit of quantification, LLOQ; Long-acting I.M. therapy, LA; Oral therapy, OR; Therapeutic drug monitoring, TDM; The International Association of Forensic Toxicologists ,TIAFT; Ultra-high performance liquid chromatography - tandem mass spectrometry, UHPLC-MS/MS; Venous dried blood spot, v-DBS;
\end{abstract}




\section{Abstract}

Background: Dried blood spot (DBS) sampling has been proposed as an alternative for venous blood collection in therapeutic drug monitoring (TDM) of antipsychotics. For implementation in routine practice, a comparison between capillary and venous blood concentrations is mandatory.

Results: A DBS method for quantification of antipsychotics was clinically validated. First, whole blood therapeutic ranges were calculated using the blood:serum ratio. Calculation of DBS:blood ratios and Passing Bablok regression analysis demonstrated that concentrations obtained by DBS analysis were highly comparable to those obtained by conventional whole blood analysis. Clinical interpretation of serum, whole blood and DBS concentrations were highly identical (sensitivity 91.6-97.6\%).

Conclusion: This is the first clinical study demonstrating the value of DBS sampling in TDM of antipsychotics.

\section{Introduction}

Psychotic symptoms, caused by schizophrenia, schizophreniform, psycho-organic or bipolar disorder, are usually treated with psychotherapy in combination with antipsychotic drugs. Although antipsychotics have proven to be essential for a good therapeutic outcome, there is a wide interpatient variability in response to therapy. Therefore, therapeutic drug monitoring (TDM) of the blood levels of antipsychotics can help in finding the right therapy, explaining non-response, pharmacokinetic interactions, poor response or eventual toxicity. TDM is recommended for almost all commonly used antipsychotics. Usually, monitoring of drugs is performed using serum or plasma obtained by classic blood withdrawal [1-5].

In a psychiatric population, the need for alternative ways of performing TDM is high, especially since blood withdrawal is often experienced as frightening or at least unpleasant. One of these alternative techniques which has gained importance in the last several years, is the use of dried blood spot (DBS) sampling. Being less invasive, easy and inexpensive, with convenient transport and storage, DBS has lots of advantages [6, 7]. So far, only a few publications on the use of DBS for measuring antipsychotic concentrations have been published. Some multi-analyte DBS-methods include one or two antipsychotics besides different other drug classes [8-10]. In 2011, a quantitative DBS method for clozapine and two metabolites using liquid chromatography (LC) coupled to electrochemical detection (ECD) was described. The method was only tested for venous blood spotted on DBS cards, while no capillary blood was collected following a finger prick [8]. In 2014, we developed a multianalyte DBS method for the analysis of 15 antipsychotics and 7 metabolites using ultra-high performance liquid chromatography - tandem mass spectrometry (UHPLC-MS/MS) [11]. Although this method was fully validated based upon EMA and FDA guidelines, a clinical validation should be 
performed before it can be implemented in routine practice. To this end, the DBS method should be evaluated on a large number of patient samples, thereby comparing the capillary concentrations with venous whole blood concentrations [12-14]. Indeed, as has been demonstrated for several compounds, one cannot simply assume that capillary blood concentrations will be the same for all compounds at all instances. In fact, capillary blood is a mixture of venous and arterial blood. Contamination from interstitial fluid, hemolysis and residual contaminations from the surface of the skin can influence capillary concentrations too [15-18]. Therefore, a thorough clinical study was conducted to make a comparison between antipsychotic concentrations found in venous whole blood and capillary DBS samples. This clinical validation consisted of an analysis of the DBS:blood ratio, statistical analysis using Passing-Bablok regression and a comparison of the clinical interpretation of the results. Two limitations should be added here. First, as the clinical validation was dependent on the collection of patient samples, not all antipsychotics which can be measured with our DBS method were present in these samples. Second, no statistical analysis was performed when the number of samples containing a specific antipsychotic was too low.

\section{Materials and methods}

\section{Patient samples}

Samples were collected from inpatients at 3 psychiatric hospitals in Belgium (Sint-Norbertus, Duffel, Belgium; Broeders Alexianen, Boechout, Belgium; Sint-Amadeus, Mortsel, Belgium). This study was approved by the Ethics Committee of the University Hospital of Antwerp (Reference 13/30/300) and all patients signed the informed consent. Inclusion criterion was a clinical diagnosis of schizophrenia, schizophreniform, schizo-affective or bipolar disorder, based on the criteria defined by DSM-IV-TR. Patients had to be in 'steady-state' condition, which means that the dose of their antipsychotic drug may not be changed in the last 7 days before blood withdrawal. For each patient, age, sex, medication scheme, time and date of sampling, time and date of the last dose of the drug and diagnosis for which the medication was prescribed were recorded. As recommended, blood was taken just prior to the morning dose (trough concentration). To make data comparable, serum, whole blood (EDTA) and capillary blood were taken at the same time point.

Capillary DBS samples (c-DBS) were obtained by finger prick. First, the finger was disinfected with a $70 \%$ isopropanol cloth, air-dried and warmed. The fingertip was then pricked by a single-use automatic lancet (Accu-Chek ${ }^{\circledast}$ Safe-T-Pro Plus, Roche Diagnostics, Mannheim, Germany). After wiping off the first drop of blood, the blood was collected into a $25 \mu \mathrm{L}$ endto-end capillary (Hirschmann Laborgeräten Eberstadt, Germany). Once entirely filled, the 
capillary was placed in the center of a marked circle on FTA ${ }^{\mathrm{TM}}$ DMPK-C Cards (GE Healthcare, Freiburg, Germany) until the capillary was completely emptied. There are two reasons for applying exactly $25 \mu \mathrm{l}$ of blood onto the DBS card. First, the hematocrit effect can largely be overcome by analyzing the whole DBS, as already described $[6,11]$. Second, a volume of $25 \mu \mathrm{l}$ was necessary to enable measuring low antipsychotic concentrations in blood. Samples were taken to the Toxicological Centre at the University of Antwerp within 4 hours. Twenty-five $\mu \mathrm{L}$ of the whole blood (EDTA) sample was also spotted on a DBS card within 6 hours after blood withdrawal, in order to make a comparison between venous whole blood on DBS (v-DBS) and c-DBS. Based upon the work of others, no stability issues are to be expected during these 6 hours $[19,20]$. DBS samples were dried for at least $3 \mathrm{~h}$ before storage at $4^{\circ} \mathrm{C}$ in zip-closure bags containing desiccant.

\section{Analytical procedures}

Serum samples were analyzed by a fully validated UHPLC-MS/MS method for quantification of 16 antipsychotics and 8 metabolites, as published before [21]. The same method was applied on whole blood samples. Sample preparation consists of a simple liquid-liquid extraction of $200 \mu \mathrm{L}$ of serum or whole blood using methyl tert-butyl ether (MTBE) as extraction solvent at $\mathrm{pH}$ 9.5, after adding an internal standard (IS) mix containing 22 deuterated IS. The upper organic layer was transferred and evaporated to dryness. Finally, the extract was reconstituted in $50 \mu \mathrm{L}$ of acetonitrile and a volume of $0.3 \mu \mathrm{L}$ was injected into the UHPLC-MS/MS.

All antipsychotic concentrations in DBS were determined using another extensively validated UHPLC-MS/MS method for the quantification of 15 antipsychotics and 7 metabolites. Olanzapine and norolanzapine could only be quantified reliably in serum and not in DBS, probably due to instability in the aqueous reconstitution solvent. Therefore, samples containing olanzapine and its metabolite were only used for comparison between serum and whole blood concentrations [11]. Briefly, after cutting the whole blood spot (containing exactly $25 \mu$ l of blood), extraction was performed using $450 \mu \mathrm{L}$ of methanol and $150 \mu \mathrm{L}$ of MTBE. An IS mix was added. After vortex mixing for $5 \mathrm{~min}$, the extract was transferred and dried under nitrogen. Samples were reconstituted in $50 \mu \mathrm{L}$ of aqueous ammonium acetate $(10 \mathrm{mM})$ /acetonitrile $(9: 1, \mathrm{v} / \mathrm{v})$ and a volume of $10 \mu \mathrm{L}$ was injected into the UHPLC-MS/MS. Instrumentation specifications were identical for both the serum and DBS method. The UHPLC-MS/MS instrument consists of an Agilent 1290 Infinity LC system (Agilent Technologies, Santa Clara, CA, USA) coupled with an Agilent 6460 Triple Quadrupole mass spectrometer (MS) run in Jetstream ${ }^{\circledR}$ electrospray ionization (ESI) mode. The LC system was 
optimized for rapid resolution using an Agilent $\mathrm{SB} \mathrm{C}_{18}$ reversed-phase column $(2.1 \times 50 \mathrm{~mm}$, $1.7 \mu \mathrm{m}$ ) (Agilent Technologies) with column oven temperature at $40^{\circ} \mathrm{C}$. The mobile phase consists of aqueous ammonium acetate $(10 \mathrm{mM})$ at $\mathrm{pH} 3.7$ (A) and acetonitrile (B) at a flow rate of $0.5 \mathrm{~mL} / \mathrm{min}$. Gradient elution was programmed as follows: starting conditions $10 \% \mathrm{~B}$; increasing to $75 \%$ B between 0 and $2.5 \mathrm{~min}$; further increase to $95 \%$ B between 2.5 and 3 $\mathrm{min}$; retain $95 \% \mathrm{~B}$ between 3 and $4.5 \mathrm{~min}$; go back to initial conditions from 4.6 to $6 \mathrm{~min}$. The MS conditions were: positive mode, nebulizer gas: nitrogen, sheath gas temperature: $400^{\circ} \mathrm{C}$, sheath gas flow: $12 \mathrm{~L} / \mathrm{min}$, nebulizer pressure: $50 \mathrm{psi}$, capillary voltage: $3000 \mathrm{~V}$, and nozzle voltage: $0 \mathrm{~V}$. Three ion transitions were monitored for each analyte using dynamic multiplereaction monitoring ( $\mathrm{dMRM}$ ) mode. Mass spectrometric conditions were identical for both the serum and DBS method as described before $[11,21]$.

\section{Data analysis}

Demographic data of study participants, calculated ratios and clinical comparison with determination of the mean values and $95 \%$ confidence intervals $(\mathrm{Cl})$ was performed using Excel.

To evaluate the correlation between venous whole blood, v-DBS and c-DBS, Passing Bablok regression analysis was performed for every compound separately using MedCalc ${ }^{\circledR}$ (MedCalc Software bvba, Ostend, Belgium).

\section{Results and discussion}

\section{Patients}

In total, 111 patients (75 male, 36 female; age range 19-65 years) were included in the clinical study. From 100 patients, serum, whole blood and v-DBS were collected. From 73 of these 100 patients, also a capillary finger prick was taken.

An overview of the demographic data per compound, together with the daily dose (DD) divided into oral (OR) and long-acting injectable therapy (LA) is presented in Table 1. Only eleven of the sixteen antipsychotics which can be detected with the serum method were found in the patient samples: amisulpride, aripiprazole, bromperidol, clozapine, haloperidol, olanzapine, paliperidone, pipamperone, quetiapine, risperidone and zuclopenthixol. The other compounds (asenapine, iloperidone, lurasidone, levosulpiride and sertindole) were not prescribed by psychiatrists in Belgium or not (yet) available on the Belgian market. For some antipsychotics, the number of patient samples was below 10 and consequently too small to perform statistical analysis. This was the case for bromperidol $(n=5)$, pipamperone $(n=4)$ and 
zuclopenthixol $(n=6)$. For the main metabolite of haloperidol, reduced haloperidol, only 3 out of the 10 patient samples showed results above the lower limit of quantification (LLOQ) in serum. Consequently, these concentrations cannot be used for statistical analysis and reduced haloperidol was not included for the clinical validation. In total, 8 antipsychotics and 4 of their metabolites could be evaluated for comparison between serum and whole blood. As olanzapine and norolanzapine were not quantified in DBS samples, 7 antipsychotics and 3 metabolites were used for the serum-whole blood-DBS comparison. For patients under LA therapy, the DD was calculated as the dose of the depot formulation divided by the number of days between 2 injections. For haloperidol, paliperidone and zuclopenthixol, there is a large difference between the OR and the LA DD. There is no difference between the OR and LA DD for olanzapine and risperidone. In some cases, antipsychotics were found which were not mentioned on medication schemes (indicated in Table 1 as NM). In total, 12 antipsychotics were found which could not be verified by the patients' medication scheme, while all identification criteria defined in the analytical method were fulfilled [21]. Since we only had data concerning the last 7 days before sample collection, we could not verify the antipsychotics taken more than a week before. Especially antipsychotics with a long half-life or long-acting formulations can be present in blood for a longer period of time.

\section{Comparison between serum and whole blood}

According to the Arbeitsgemeinschaft für Neuropsychopharmakologie und Pharmakopsychiatrie (AGNP) Consensus Guidelines for TDM in Psychiatry, measuring antipsychotic concentrations is preferable in serum or plasma, while whole blood is 'abandoned' for TDM. The most important reasons for preferring serum or plasma over whole blood are the ease of chemical analysis and storage, and the homogeneity of plasma and serum compared to clotted blood. The difference between serum and plasma would be minimal, but has not been extensively evaluated in literature. In comparison with serum, plasma contains clotting factors and an anticoagulant which is present in the collection tubes (e.g. EDTA), while the concentration of binding proteins is assumed to be similar $[1,14,22$, 23]. In routine practice, serum and plasma can both be used for TDM purposes. Due to the enormous interest in DBS as an alternative for TDM, we should be aware of the differences between serum and whole blood, either collected by classical venous blood sampling or by finger prick. Indeed, concentrations in whole blood can differ from those in serum. Drugs can bind to constituents in both serum and/or in blood cells. The blood cell membrane acts as a barrier which can slow down or even impede movement of a compound into blood cells. For example, some drugs do not bind to plasma proteins, which makes plasma but also whole 
blood useful for measuring drug concentrations. On the other hand, some drugs are too large or too polar to enter cells which means that blood cells only act as diluent for plasma. Other drugs freely enter blood cells but do not bind to constituents or plasma proteins, meaning that blood values can be used without the risk for misinterpretation. However, most drugs do bind to plasma proteins, and enter and bind to blood cells [14, 16, 23]. Most antipsychotics are basic drugs which are highly bound to plasma proteins [24]. For example, protein binding of clozapine is known to be $95 \%$ with main binding to $\alpha_{1}$-acid glycoprotein $[22,25]$. For this reason, the relationship between serum or plasma and whole blood has to be determined. Therefore, before we were able to evaluate our DBS results, we compared the antipsychotic concentrations in serum and whole blood, both analyzed with the conventional method. This allowed us to determine the blood:serum ratio for the 8 antipsychotics and 4 metabolites which were found in the patient samples. A mean ratio together with the $95 \% \mathrm{Cl}$ was calculated and compared with literature $[22,25,26]$. Overall, the $95 \% \mathrm{Cl}$ of the calculated ratios of most of the antipsychotics was narrow and included the ratio mentioned in Baselt R.C. (Table 2). For the metabolites norolanzapine, $70 \mathrm{H}-\mathrm{N}$-desalkylquetiapine and $7 \mathrm{OH}-$ quetiapine, the blood:plasma(serum) ratio could not be compared with literature since this is the first time blood:serum ratios were determined for these metabolites. Therefore, these data are highly valuable but should be confirmed by others. Only for amisulpride, clozapine and paliperidone, the blood:plasma ratio reported in Baselt R.C. was just not included in our calculated $95 \% \mathrm{Cl}$ (amisulpride calculated blood:serum ratio 1.2 vs. published blood:plasma ratio 1.4 ; clozapine 1.0 vs. 0.8 ; paliperidone 0.7 vs. 0.8 ] [25]. However, for clozapine two other articles described a blood:plasma ratio of 0.8 (range 0.5-1.0, $n=29)$ and $0.9(n=45)$, respectively, which was more comparable with our results $[22,26]$. Moreover, Fisher et al. reported a blood:plasma ratio for paliperidone of 0.7 (range $0.6-0.9, n=37$ ) which was identical to our results [26]. The ratios determined by these two articles are considered reliable since they were based on an acceptable number of concentrations. Therefore, we feel that our calculated ratios for clozapine and paliperidone can be considered reliable. Only for amisulpride, Fisher et al. described a ratio between 1.2 and 1.7 based on only 2 patient samples, which makes it difficult to draw conclusions. For olanzapine, the difference was even more pronounced (calculated blood:serum ratio 1.2 vs. published blood:plasma ratio 0.6) [25]. Olanzapine is known to be a difficult compound with frequently reported instability in an aqueous environment $[11,27,28]$. However, as we previously demonstrated in our extensively validated serum method, olanzapine can be quantified reliably without instability problems when no water was used to reconstitute the extract before analysis [21]. Still, it is known that olanzapine is more unstable in whole blood than in serum or plasma $[20,27,29]$. 
Not only the matrix type but also the sample preparation, with or without the use of water, will influence olanzapine stability and thus also the blood:serum ratio. Therefore, it is difficult to compare the calculated blood:serum ratio with the previously published data. As was already mentioned, olanzapine was not included in our DBS method. Still, the difference in ratios should be a warning for the difficult quantification of olanzapine which is highly influenced by instability problems in matrices and during sample preparation.

The blood:serum ratio is useful as it can provide information on how drugs are distributed between serum and whole blood. For instance, some drugs with high affinity for plasma proteins do not partition into erythrocytes meaning that blood cells act as a diluent. For these compounds, a ratio between $0.55-0.60$ is seen. The influence of the hematocrit is the only thing that has to be taken into account when whole blood is used. Since most of the psychiatric patients are not suffering from serious somatic illnesses, we can assume that the hematocrit is fairly constant. For other drugs a ratio of one is seen because these drugs do enter erythrocytes but do not bind to proteins there or in plasma. For drugs that enter blood cells and bind both there and in plasma, the influence of the unbound fraction in plasma and the blood cell partitioning has to be taken into account. The higher the ratio, especially $>2$, the more the variability in blood cell affinity will become a critical factor [16, 23].

Overall, the obtained blood:serum ratios, which were comparable with literature, allow us to derive serum concentrations from whole blood measurements, except for olanzapine. Alternatively, these ratios can be used to calculate whole blood therapeutic reference ranges, starting from the therapeutic ranges defined by TIAFT and AGNP-TDM guidelines (Table 3). When aiming at implementing DBS analysis in routine practice, the approach of using blood therapeutic ranges may be more straightforward and convenient than sticking to the serum (plasma) therapeutic ranges and applying a correction factor for every measurement. For $7 \mathrm{OH}-\mathrm{N}$-desalkylquetiapine, no therapeutic reference range in serum is defined [1]. As was mentioned, for norclozapine and $7 \mathrm{OH}$-quetiapine, the blood:serum ratio could not be compared with literature. For amisulpride, a small difference was seen with the blood:plasma ratio in Baselt, but not with the limited data published by Fisher et al. $[25,26]$. Although we acknowledge that even larger datasets may lead to a further refinement, overall, we considered the calculated whole blood therapeutic reference ranges to be sufficiently reliable for our purpose, i.e. for the interpretation of the results in the context of a clinical comparison.

\section{Comparison between venous whole blood and DBS}


Before comparing c-DBS concentrations of antipsychotics with serum concentrations, two aspects need to be evaluated. First, concentrations obtained by DBS analysis should be comparable with whole blood concentrations measured by the conventional method. Second, it should be evaluated whether concentrations in capillary and venous blood are similar. This can be done by comparing v-DBS and c-DBS.

Two kinds of analysis were performed on 7 antipsychotics and 3 metabolites to evaluate the equivalence of concentrations in whole blood and v-DBS, in whole blood and c-DBS, and in cDBS and v-DBS. First, we calculated mean ratios of v-DBS versus whole blood, of c-DBS versus whole blood, and of c-DBS versus v-DBS. Concentrations can be considered equivalent if the $95 \% \mathrm{Cl}$ of their ratio includes 1 . In that case, the influence of analyzing whole blood as v-DBS or as c-DBS can be considered as minimal. Additionally, as recently described in preliminary guidelines defined by the Alternative Sampling Strategies Committee of IATDMCT (International Association of TDM and Clinical Toxicology), we performed Passing-Bablok regression analysis to evaluate agreement between the two analytical methods, including a possible bias that may be present [13]. There is no proportional difference between the two methods if the $95 \% \mathrm{Cl}$ of the slope contains 1 . On the other hand, if the $95 \% \mathrm{Cl}$ of the intercept includes 0 , there is no systematic difference. Both criteria must be fulfilled to conclude that the conventional and DBS results are comparable [13, 30-32].

As can be seen in Table 2, the $95 \% \mathrm{Cl}$ of the v-DBS:whole blood ratio was narrow for all compounds and for 8 antipsychotics the $\mathrm{Cl}$ included 1 . Only for clozapine and 7OH-Ndesalkylquetiapine, the $95 \% \mathrm{Cl}$ does not contain 1, but the difference remained limited (ratio of 1.1-1.4 for clozapine, 1.1-1.5 for 7OH-N-desalkylquetiapine). Passing-Bablok analysis to compare concentrations in v-DBS and whole blood did not demonstrate systematic or proportional differences for all but one of the antipsychotics (Table 4). Only for quetiapine the $95 \% \mathrm{Cl}$ of the slope did not contain 1 (95\% $\mathrm{Cl} 1.09-1.22)$, meaning that there is a proportional difference between the two methods.

For the comparison between venous whole blood and c-DBS, the $95 \% \mathrm{Cl}$ of the c-DBS:whole blood ratio contained 1 for 7 compounds. For haloperidol, only 2 patient samples were available, so no conclusion could be drawn. For amisulpride and paliperidone, the $95 \% \mathrm{Cl}$ did not include 1 (ratio of 1.2-1.4 for amisulpride, 1.1-1.2 for paliperidone). Passing-Bablok analysis showed no proportional difference for all antipsychotics except for amisulpride (95\% $\mathrm{Cl} 1.12-1.94)$, quetiapine (95\% Cl 1.02-1.14), $7 \mathrm{OH}-\mathrm{N}$-desalkylquetiapine (95\% Cl 1.06-2.98) and paliperidone (95\% Cl 1.06-1.20) (Table 5). A systematic difference was only seen for quetiapine $(95 \% \mathrm{Cl}-6.02--1.15)$ and $70 \mathrm{H}-\mathrm{N}$-desalkylquetiapine $(95 \% \mathrm{Cl}-9.79--0.48)$. 
Similarly, for 9 compounds the $95 \% \mathrm{Cl}$ of the c-DBS:v-DBS ratio includes 1 (Table 2), meaning that there is no difference between capillary and venous blood analyzed on DBS cards. Only for haloperidol, no conclusions can be drawn, since only 2 patient samples were available. Passing-Bablok analysis showed aberrant results for quetiapine and paliperidone, since for these two compounds the $95 \% \mathrm{Cl}$ of the slope did not contain 1 (95\% Cl of 0.91-0.99 for quetiapine, 1.01-1.13 for paliperidone). However, as can be seen in Table 6, the differences are only minimal.

The differences observed for some compounds, as presented in Tables 2, 4, 5 and 6, need to be confirmed with larger datasets. However, this was beyond the scope of this proof-ofconcept study. Overall, our results do suggest that for most evaluated compounds capillary and venous concentrations determined by DBS analysis are largely comparable with venous blood analyzed by the conventional method. Considering that capillary blood consists of both venous and arterial blood, usually no pronounced differences are seen between venous and capillary concentrations once a distribution equilibrium is achieved [16, 23]. Since our patients are in steady-state condition, it is to be expected that this equilibrium is reached. The previously calculated blood:serum ratios can be used to convert DBS results to serum concentrations for which therapeutic reference ranges have been defined $[1,33,34]$.

\section{Clinical comparison}

Another important issue is the clinical interpretation of the obtained blood levels. As mentioned above, therapeutic ranges have been defined for the antipsychotics in serum or plasma [1]. In a TDM context, results are reported in relation to this therapeutic range. Clinicians want to know if the blood level is below, within or above the defined therapeutic reference range to make decisions on dosing, therapy change, duration, etc. Yet, it should be noticed that a therapeutic range is population-based and is not necessarily applicable to every patient. Hence, blood levels should always be interpreted in a clinical context. Some patients may show a good response below the lower limit of the therapeutic range, while others need much higher blood levels. Anyway, clinical interpretations should be the same for both the conventional and DBS method.

A comparison between the conventional serum, whole blood and DBS method was performed based on the clinical interpretation. Therefore, the whole blood and DBS concentrations were interpreted using the calculated whole blood therapeutic ranges (Table 3), while for the serum concentrations the serum therapeutic ranges defined by the AGNPTDM and The International Association of Forensics Toxicologists (TIAFT) guidelines were used [1]. Serum and blood levels were divided into 5 groups: not detected, below the LLOQ, 
below the therapeutic range, within the therapeutic range or above therapeutic range. Afterwards, results derived from venous whole blood analysis were compared with v-DBS and c-DBS data. For some metabolites, like reduced haloperidol and $7 \mathrm{OH}-\mathrm{N}-$ desalkylquetiapine, a therapeutic range is lacking in literature. Therefore, data from these two metabolites were not included. Results are summarized in Table 7. Sensitivity was calculated by dividing the number of identical interpretations (sum of '< LLOQ', 'below, within and above therapeutic range') by the total number of interpretations. For the comparison between the conventional venous method and v-DBS, a sensitivity of 92.2\% was achieved while for the comparison between venous blood and c-DBS and v-DBS and c-DBS, the sensitivity was even higher (95.8\% and 97.6\%, respectively). For the compounds with a different interpretation, concentrations were always close to the lower or upper limits of the defined ranges, with limited nominal differences between the concentrations measured in the distinct matrices. This means that there was never an important difference in interpretation, for example one concentration below and the other above the therapeutic range. Two remarks should be taken into consideration. In two out of the 37 samples containing $7 \mathrm{OH}$-quetiapine, the difference appeared to be larger since the venous concentration was within the therapeutic range (calculated venous concentration: $1.0 \mathrm{ng} / \mathrm{ml}$ and $1.4 \mathrm{ng} / \mathrm{ml}$ ) while the DBS concentrations were below LLOQ. However, the LLOQ of $70 \mathrm{H}$-quetiapine was $1 \mathrm{ng} / \mathrm{mL}$ and the therapeutic range was defined as 1-48 ng/mL, meaning that the difference between the concentrations was only minimal. In three other cases, an antipsychotic was detected with one method (concentration < LLOQ) while it was not detected with the other method. This was seen for two samples containing $70 \mathrm{H}-$ quetiapine (sample 1: conventional method < LLOQ, v-DBS and c-DBS not detected; sample 2: conventional method not detected, v-DBS $<$ LLOQ) and for 1 out of 18 samples containing risperidone (conventional method not detected, v-DBS and c-DBS $<$ LLOQ).

Finally, serum results were also compared with c-DBS results. In the future, the concentrations found in these two matrices are preferentially interchangeable, meaning that the interpretation of serum and c-DBS levels should be identical. Comparison of the clinical interpretation of serum and c-DBS data resulted in a high sensitivity of $91.6 \%$. As for the other comparisons, concentrations of compounds with a different interpretation were always close to the lower or upper limits of the defined ranges, with only limited differences between measured concentrations. Similarly, for one sample containing $7 \mathrm{OH}$-quetiapine, the serum concentration $(1.4 \mathrm{ng} / \mathrm{ml})$ was within the therapeutic range while the c-DBS result was below LLOQ (1 ng/ml). Still, the nominal difference between those two concentrations was limited and would not influence clinical decision making. 
The non-concordancy of amisulpride, paliperidone and quetiapine concentrations, as suggested by the analysis of the DBS:blood ratios and Passing-Bablok analysis (cfr. Tables 2, 4, 5 and 6), were not confirmed in this section. A possible explanation is that the small nominal differences that were found with the statistical analyses will only have a limited influence on the final concentrations and thus will not influence the clinical interpretation. Overall, we can state that clinical interpretation of the DBS concentrations will result in a reliable conclusion, which will be almost identical to the interpretation of the venous blood or serum concentrations. Only for the $\mathbf{7 0 H}$-metabolite of quetiapine, the small difference between the therapeutic range $(1-48 \mathrm{ng} / \mathrm{ml})$ and the LLOQ $(1 \mathrm{ng} / \mathrm{ml})$ should be kept in mind and should be reported to the clinician in order to make correct interpretation of the results.

\section{Conclusion}

This is the first clinical study to evaluate the use of DBS sampling for TDM of antipsychotics. A thorough clinical validation of the DBS method for quantification of commonly used antipsychotics demonstrated that DBS sampling is a promising alternative for classical blood sampling and can be used in routine practice. Since therapeutic ranges defined for serum cannot be used for interpretation of whole blood concentrations (both venous and capillary), whole blood therapeutic ranges were calculated from the serum therapeutic range using the blood:serum ratio.

Calculation of the v-DBS:blood, c-DBS:blood and c-DBS:v-DBS ratio, together with Passing Bablok regression analysis demonstrated that concentrations obtained by DBS analysis were highly comparable with those obtained by conventional whole blood analysis. Only for quetiapine, the criteria defined for Passing-Bablok regression analysis were not fulfilled. However, these apparent non-concordances did not influence the clinical interpretation of the results. Clinical interpretation of the serum, whole blood and DBS concentrations using the therapeutic ranges was highly comparable (sensitivity of $92.2 \%$ for venous vs. v-DBS, $95.8 \%$ for venous vs. c-DBS, $97.6 \%$ for v-DBS vs. c-DBS, and $91.6 \%$ for serum vs. c-DBS). When the interpretation between concentrations analyzed by the two analytical methods was different, this difference was always minimal and related to concentrations close to the upper or lower limit of the defined ranges.

\section{Future perspective}

DBS sampling is a highly promising technique for TDM of different therapeutic drugs like antidepressants, immunosuppressants, antibiotics and antipsychotics. However, literature about the 
clinical validation with comparison between venous and capillary blood concentrations is scarce.

While most published methods are extensively validated in accordance with the analytical guidelines, little attention has been given to the clinical part [14]. Only recently, some guidelines were suggested by the Alternative Sampling Strategies Committee of the International Association of Therapeutic Drug Monitoring and Clinical Toxicology (IATDMCT) [13]. In the future, the development of a DBS method should always include both an analytical and a clinical validation before these methods can be implemented in a routine laboratory.

\section{References}

1. Hiemke C, Baumann P, Bergemann N et al.: AGNP Consensus Guidelines for Therapeutic Drug Monitoring in Psychiatry: Update 2011. Pharmacopsychiatry 44(6), 195-235 (2011).

2. Keefe RS, Bilder RM, Davis SM et al.: Neurocognitive effects of antipsychotic medications in patients with chronic schizophrenia in the CATIE Trial. Arch Gen Psychiatry 64(6), 633-647 (2007).

3. Mishara AL, Goldberg TE: A meta-analysis and critical review of the effects of conventional neuroleptic treatment on cognition in schizophrenia: opening a closed book. Biol Psychiat 55(10), 1013-1022 (2004).

4. Tarr GP, Glue P, Herbison P: Comparative efficacy and acceptability of mood stabilizer and second generation antipsychotic monotherapy for acute mania--a systematic review and meta-analysis. J Affect Disorders 134(1-3), 14-19 (2011).

5. Vieta E, Locklear J, Gunther $\mathrm{O}$ et al.: Treatment options for bipolar depression: a systematic review of randomized, controlled trials. J Clin Psychopharm 30(5), 579-590 (2010).

6. De Kesel PM, Sadones N, Capiau S, Lambert WE, Stove CP: Hemato-critical issues in quantitative analysis of dried blood spots: challenges and solutions. Bioanalysis 5(16), 20232041 (2013).

7. Stove $\mathrm{CP}$, Ingels AS, De Kesel PM, Lambert WE: Dried blood spots in toxicology: from the cradle to the grave? Crit. Rev. Toxicol. 42(3), 230-243 (2012).

8. Saracino MA, Lazzara G, Prugnoli B, Raggi MA: Rapid assays of clozapine and its metabolites in dried blood spots by liquid chromatography and microextraction by packed sorbent procedure. J Chromatogr A 1218(16), 2153-2159 (2011).

9. Temesi D, Swales J, Keene W, Dick S: The stability of amitriptyline N-oxide and clozapine Noxide on treated and untreated dry blood spot cards. J Pharm Biomed Anal 76, 164-168 (2013).

10. Versace F, Deglon J, Lauer E, Mangin P, Staub C: Automated DBS extraction prior to HILIC/RP LC-MS/MS target screening of drugs. Chromatographia 76, 1281-1293 (2013). 
11. Patteet L, Maudens KE, Stove CP et al.: The use of dried blood spots for quantification of 15 antipsychotics and 7 metabolites with ultra-high performance liquid chromatography tandem mass spectrometry. Drug testing and analysis (DOI: 10.1002/dta.1698), (2014).

12. Sadones N, Archer JR, Ingels AS et al.: Do capillary dried blood spot concentrations of gamma-hydroxybutyric acid mirror those in venous blood? A comparative study. Drug Test Anal 7(4), 336-340 (2015).

13. Alffenaar JW: Dried blood spot analysis for therapeutic drug monitoring: clinical validation precedes implementation. IATDMCT Compass, 5-6 (September 2014).

14. Wilhelm AJ, Den Burger JC, Swart EL: Therapeutic drug monitoring by dried blood spot: progress to date and future directions. Clin Pharmacokinet 53(11), 961-973 (2014).

15. Bush V: Effects of pre-analytical variables in therapeutic drug monitoring. In: Therapeutic drug monitoring: newer drugs and biomarkers, Dasgupta A (Ed.), Elsevier Inc., London, UK, 31-48 (2012).

16. Emmons G, Rowland M: Pharmacokinetic considerations as to when to use dried blood spot sampling. Bioanalysis 2(11), 1791-1796 (2010).

17. Mohammed BS, Cameron GA, Cameron L, Hawksworth GH, Helms PJ, Mclay JS: Can fingerprick sampling replace venous sampling to determine the pharmacokinetic profile of oral paracetamol? Br J Clin Pharmacol 70(1), 52-56 (2010).

18. De Kesel PM, Lambert WE, Stove CP: Why dried blood spots are an ideal tool for CYP1A2 phenotyping. Clin Pharmacokinet 53(8), 763-771 (2014).

19. Fisher DS, Partridge SJ, Handley SA, Flanagan RJ: Stability of some atypical antipsychotics in human plasma, haemolysed whole blood, oral fluid, human serum and calf serum. Forensic Sci Int 229(1-3), 151-156 (2013).

20. Saar E, Gerostamoulos D, Drummer OH, Beyer J: Assessment of the stability of 30 antipsychotic drugs in stored blood specimens. Forensic Sci Int 215(1-3), 152-158 (2012).

21. Patteet L, Maudens KE, Sabbe B, Morrens M, De Doncker M, Neels H: High throughput identification and quantification of 16 antipsychotics and 8 major metabolites in serum using ultra-high performance liquid chromatography-tandem mass spectrometry. Clin Chim Acta 429, 51-58 (2014).

22. Flanagan RJ, Yusufi B, Barnes TR: Comparability of whole-blood and plasma clozapine and norclozapine concentrations. Br J Clin Pharmacol 56(1), 135-138 (2003).

23. Rowland $\mathrm{M}$, Emmons $\mathrm{GT}$ : Use of dried blood spots in drug development: pharmacokinetic considerations. The AAPS journal 12(3), 290-293 (2010).

24. Patteet L, Morrens M, Maudens KE, Niemegeers P, Sabbe B, Neels H: Therapeutic drug monitoring of common antipsychotics. Ther Drug Monit 34(6), 629-651 (2012).

25. Baselt RC. Disposition of toxic drugs and chemicals in man: tenth edition. Biomedical Publications, Seal Beach, California, USA (2014).

26. Fisher DS, Partridge SJ, Handley SA, Couchman L, Morgan PE, Flanagan RJ: LC-MS/MS of some atypical antipsychotics in human plasma, serum, oral fluid and haemolysed whole blood. Forensic Sci Int 229(1-3), 145-150 (2013).

27. Berna M, Ackermann B, Ruterbories K, Glass S: Determination of olanzapine in human blood by liquid chromatography-tandem mass spectrometry. J Chromatogr B 767(1), 163-168 (2002).

28. Olesen OV, Linnet K: Determination of olanzapine in serum by high-performance liquid chromatography using ultraviolet detection considering the easy oxidability of the compound and the presence of other psychotropic drugs. J Chromatogr B 714(2), 309-315 (1998).

29. Fisher DS, Van Schalkwyk GI, Seedat S, Curran SR, Flanagan RJ: Plasma, oral fluid, and wholeblood distribution of antipsychotics and metabolites in clinical samples. Ther Drug Monit 35(3), 345-351 (2013).

30. Sadones N, Archer JR, Ingels AM et al.: Do capillary dried blood spot concentrations of gamma-hydroxybutyric acid mirror those in venous blood? A comparative study. Drug Test Anal, (2015). 
31. Passing $\mathrm{H}$, Bablok: A new biometrical procedure for testing the equality of measurements from two different analytical methods. Application of linear regression procedures for method comparison studies in clinical chemistry, Part I. Journal of clinical chemistry and clinical biochemistry. Zeitschrift fur klinische Chemie und klinische Biochemie 21(11), 709-720 (1983).

32. Passing $\mathrm{H}$, Bablok $\mathrm{W}$ : Comparison of several regression procedures for method comparison studies and determination of sample sizes. Application of linear regression procedures for method comparison studies in Clinical Chemistry, Part II. Journal of clinical chemistry and clinical biochemistry. Zeitschrift fur klinische Chemie und klinische Biochemie 22(6), 431-445 (1984).

33. Edelbroek PM, Van Der Heijden J, Stolk LM: Dried blood spot methods in therapeutic drug monitoring: methods, assays, and pitfalls. Ther Drug Monit 31(3), 327-336 (2009).

34. Ji QC, Liu G, D'arienzo CJ, Olah TV, Arnold ME: What is next for dried blood spots? Bioanalysis 4(16), 2059-2065 (2012).

\section{Reference annotations}

\section{Ref. 1}

** The most complete and extensive guidelines for TDM of drugs important for psychiatry. All aspects of TDM, including the importance of detecting metabolites and genetic testing are described.

\section{Ref. 11}

* First multi-analyte method for quantification of antipsychotics in dried blood spots.

\section{Ref. 16}

** Pharmacokinetic issues concerning the use of DBS including a first concept about the comparison of DBS and plasma measurements. 
Table 1: Patient demographics and daily dose by analyte.

\begin{tabular}{|c|c|c|c|c|c|c|c|c|c|c|c|}
\hline \multirow[b]{2}{*}{ Analyte } & \multirow[b]{2}{*}{ Sex } & \multirow[b]{2}{*}{$\mathbf{n}$} & \multirow{2}{*}{$\begin{array}{l}\text { Serum \& venous } \\
\text { No. of samples }\end{array}$} & \multirow{2}{*}{$\begin{array}{l}\text { DBS } \\
\text { No. of samples }\end{array}$} & & \multirow[b]{2}{*}{ OR/LA/NM ${ }^{a}(n)$} & \multicolumn{3}{|c|}{ Dose (mg/day) } \\
\hline & & & & & Median & Min & Max & & Median & Min & Max \\
\hline \multirow[t]{2}{*}{ Amisulpride } & Male & 10 & \multirow[t]{2}{*}{12} & \multirow[t]{2}{*}{11} & \multirow[t]{2}{*}{47} & \multirow[t]{2}{*}{27} & \multirow[t]{2}{*}{56} & \multirow[t]{2}{*}{ OR (12) } & \multirow[t]{2}{*}{500} & \multirow[t]{2}{*}{200} & \multirow[t]{2}{*}{1200} \\
\hline & Female & 2 & & & & & & & & & \\
\hline \multirow[t]{2}{*}{ Aripiprazole } & Male & 9 & \multirow[t]{2}{*}{16} & \multirow[t]{2}{*}{14} & \multirow[t]{2}{*}{38} & \multirow[t]{2}{*}{21} & \multirow[t]{2}{*}{55} & OR $(15)^{*}$ & \multirow[t]{2}{*}{15} & \multirow[t]{2}{*}{7.5} & \multirow[t]{2}{*}{30} \\
\hline & Female & 7 & & & & & & $\operatorname{NM}(1)^{\mathrm{a}}$ & & & \\
\hline \multirow[t]{2}{*}{ Bromperidol } & Male & 2 & \multirow[t]{2}{*}{5} & 5 & 52 & 36 & 53 & $\mathrm{LA}(4)$ & 3.6 & 2.4 & 4.4 \\
\hline & Female & 1 & & & & & & & & & \\
\hline Clozapine & Male & 19 & 20 & 14 & 31 & 22 & 64 & OR (21) & 300 & 25 & 700 \\
\hline & Female & 2 & & & & & & & & & \\
\hline Haloperidol & Male & 10 & 10 & 2 & 27 & 24 & 56 & OR (3) & 11.2 & 7.5 & 15 \\
\hline & Female & 1 & & & & & & LA (7) & 3.6 & 2.7 & 4.8 \\
\hline & & & & & & & & $\operatorname{NM}(1)^{\mathrm{a}}$ & & & \\
\hline Olanzapine & Male & 20 & 30 & $\mathrm{NT}^{\circ}$ & 39 & 19 & 59 & OR (22) & 17.5 & 5 & 40 \\
\hline & Female & 9 & & & & & & LA (6) & 17.9 & 11.6 & 51.4 \\
\hline & & & & & & & & $\operatorname{NM}(2)^{a}$ & & & \\
\hline Paliperidone & Male & 21 & 35 & 26 & 36 & 20 & 64 & OR (9) & 9 & 6 & 9 \\
\hline & Female & 13 & & & & & & LA (23) & 3.6 & 1.8 & 14.3 \\
\hline & & & & & & & & $\operatorname{NM}(3)^{a}$ & & & \\
\hline Pipamperone & Male & 3 & 3 & 1 & 41 & 36 & 45 & OR (2) & - & 40 & 80 \\
\hline & Female & 0 & & & & & & $\operatorname{NM}(1)^{a}$ & & & \\
\hline Quetiapine & Male & 22 & 41 & 28 & 38 & 21 & 58 & OR (41) & 200 & 25 & 1400 \\
\hline & Female & 16 & & & & & & $\operatorname{NM}(2)^{a}$ & & & \\
\hline Risperidone & Male & 12 & 19 & 15 & 43 & 21 & 65 & OR (8) & 3.5 & 1 & 6 \\
\hline & Female & 6 & & & & & & LA (9) & 3.6 & 2.7 & 5.8 \\
\hline & & & & & & & & $\operatorname{NM}(2)^{a}$ & & & \\
\hline Zuclopenthixol & Male & 3 & 6 & 6 & 42 & 30 & 54 & OR (4) & 30 & 15 & 50 \\
\hline & Female & 3 & & & & & & LA (5) & 16.7 & 9.5 & 33.3 \\
\hline
\end{tabular}

${ }^{\circ} \mathrm{NT}$ : Olanzapine was not included in the DBS method

${ }^{a} \mathrm{NM}$ : The antipsychotic found in ' $n$ ' samples was not mentioned on the medication scheme

DBS, dried blood spots; OR, oral therapy; LA, long-acting injectable therapy;

Table 2 : Overview of the calculated blood:serum ratio (mean, 95\% confidence interval), venous blood spotted on DBS(v-DBS) versus blood ratio, capillary blood spotted on DBS (c-DBS) versus blood ratio and c-DBS versus vDBS ratio. The $95 \%$ confidence intervals $(\mathrm{Cl})$ that do not contain 1 for the $v$-DBS:blood ratio, c-DBS:blood ratio and c-DBS: v-DBS ratio are marked in bold. 


\begin{tabular}{|c|c|c|c|c|c|c|c|c|c|c|c|c|}
\hline \multirow[t]{2}{*}{ Antipsychotic } & \multicolumn{3}{|c|}{ blood:serum ratio } & \multicolumn{3}{|c|}{ v-DBS:blood ratio } & \multicolumn{3}{|c|}{$\begin{array}{l}\text { c-DBS:blood } \\
\text { ratio } \\
\text { n mean } 95 \%\end{array}$} & \multicolumn{3}{|c|}{ c-DBS:v-DBS ratio } \\
\hline & $\mathrm{n}$ & mean & $95 \% \mathrm{Cl}$ & $n$ & mean & $95 \% \mathrm{Cl}$ & & & $\mathrm{Cl}$ & $\mathbf{n}$ & mean & $95 \% \mathrm{Cl}$ \\
\hline Amisulpride & 12 & 1.2 & $1.1-1.2$ & 11 & 1.1 & $1.0-1.1$ & 8 & 1.3 & $\begin{array}{l}1.2- \\
1.4 \\
1.0-\end{array}$ & 8 & 1.2 & $1.0-1.3$ \\
\hline Aripiprazole & 16 & 0.7 & $0.6-0.7$ & 16 & 1.0 & $0.9-1.1$ & 14 & 1.0 & $\begin{array}{l}1.1 \\
1.0-\end{array}$ & 14 & 1.0 & $1.0-1.1$ \\
\hline Clozapine & 16 & 1.0 & $0.9-1.0$ & 17 & 1.2 & 1.1-1.4 & 10 & 1.1 & $\begin{array}{l}1.1 \\
0.9-\end{array}$ & 10 & 1.0 & $1.0-1.1$ \\
\hline Norclozapine & 20 & 1.4 & $1.3-1.5$ & 20 & 0.9 & $0.9-1.0$ & 12 & 1.0 & $\begin{array}{l}1.1 \\
0.1-\end{array}$ & 12 & 1.1 & $1.0-1.1$ \\
\hline Haloperidol & 11 & 0.8 & $0.7-1.0$ & 10 & 0.9 & $0.8-1.0$ & 2 & 1.6 & 3.2 & 2 & 1.6 & $0.5-2.6$ \\
\hline Olanzapine & 29 & 1.2 & $1.1-1.3$ & & & & & & & & & \\
\hline Norolanzapine & 26 & 2.2 & $1.9-2.4$ & & & & & & 1.1- & & & \\
\hline Paliperidone & 48 & 0.7 & $0.7-0.7$ & 47 & 1.0 & $1.0-1.1$ & 36 & 1.1 & $\begin{array}{l}1.2 \\
1.0-\end{array}$ & 36 & 1.1 & $1.0-1.1$ \\
\hline Quetiapine & 36 & 0.7 & $0.7-0.7$ & 34 & 1.1 & $1.0-1.2$ & 26 & 1.0 & $\begin{array}{l}1.1 \\
0.9-\end{array}$ & 26 & 1.0 & $0.9-1.1$ \\
\hline 7OH-N-desalkylquetiapine & 37 & 1.1 & $1.0-1.2$ & 32 & 1.3 & 1.1-1.5 & 25 & 1.5 & $\begin{array}{l}2.0 \\
0.8-\end{array}$ & 23 & 1.0 & $0.9-1.2$ \\
\hline 7OH-quetiapine & 36 & 1.1 & $1.0-1.1$ & 31 & 1.2 & $1.0-1.3$ & 23 & 1.0 & $\begin{array}{l}1.2 \\
1.0-\end{array}$ & 22 & 0.9 & $0.8-1.0$ \\
\hline Risperidone & 16 & 0.6 & $0.6-0.7$ & 15 & 1.0 & $1.0-1.1$ & 13 & 1.1 & 1.1 & 13 & 1.1 & $1.0-1.1$ \\
\hline
\end{tabular}

Table

3: Serum reference ranges from literature and calculated whole blood reference ranges using the blood:serum ratio.

\begin{tabular}{|c|c|c|}
\hline Antipsychotic & $\begin{array}{l}\text { Serum therapeutic } \\
\text { reference range* } \\
(\mathrm{ng} / \mathrm{ml})\end{array}$ & $\begin{array}{l}\text { Whole blood therapeutic } \\
\text { reference range } \\
\text { (ng/ml) }\end{array}$ \\
\hline Amisulpride & $100-400$ & $120-480$ \\
\hline Aripiprazole & $150-500$ & $105-350$ \\
\hline Clozapine & $350-600$ & $350-600$ \\
\hline Norclozapine & $100-600$ & $140-840$ \\
\hline Haloperidol & $1-10$ & $1-8$ \\
\hline Paliperidone & $12-60$ & $8-42$ \\
\hline Quetiapine & $75-500$ & $53-350$ \\
\hline 7OH-quetiapine & $1-48$ & $1-53$ \\
\hline Risperidone & $4-30$ & $3-21$ \\
\hline
\end{tabular}

* Based on TIAFT and AGNP-TDM guidelines (Hiemke et al. 2011)

Table 4: Results of Passing Bablok regression analysis for the comparison between venous whole blood and v-DBS. The $95 \%$ confidence intervals $(\mathrm{Cl})$ of the slope that do not contain 1 and $95 \% \mathrm{Cl}$ of the intercept that did not contain 0 are marked in bold. 


\begin{tabular}{lccccc} 
Analyte & No of samples & Slope & $\mathbf{9 5 \%} \mathbf{~ C l}$ & Intercept & $\mathbf{9 5 \% ~ C l}$ \\
\hline Amisulpride & 11 & 1.10 & $0.87-1.23$ & 2.32 & $-30.55-42.25$ \\
Aripiprazole & 16 & 1.07 & $0.97-1.17$ & -8.55 & $-26.78-2.39$ \\
Clozapine & 17 & 1.05 & $0.93-1.34$ & 1.64 & $-22.11-34.33$ \\
Norclozapine & 20 & 0.90 & $0.83-1.04$ & -0.93 & $-16.77-12.39$ \\
Haloperidol & 10 & 1.02 & $0.73-2.50$ & -0.13 & $-2.57-0.29$ \\
Paliperidone & 47 & 1.03 & $0.98-1.08$ & -0.17 & $-1.30-0.50$ \\
Quetiapine & 34 & 1.15 & $1.09-1.22$ & -3.21 & $-5.13-1.22$ \\
7OH-N-desalkylquetiapine & 32 & 1.26 & $0.95-1.93$ & -0.81 & $-4.02-0.81$ \\
7OH-quetiapine & 31 & 1.13 & $0.87-1.49$ & -0.16 & $-1.33-1.18$ \\
Risperidone & 15 & 1.04 & $0.98-1.08$ & -0.08 & $-0.44-0.12$
\end{tabular}

Table 5: Result of Passing Bablok regression analysis for the comparison between venous whole blood and c-DBS. The $95 \%$ confidence intervals $(\mathrm{Cl})$ of the slope that do not contain 1 and $95 \% \mathrm{Cl}$ of the intercept that did not contain 0 are marked in bold.

\begin{tabular}{lccccc} 
Analyte & No of samples & Slope & $\mathbf{9 5 \%} \mathbf{C l}$ & Intercept & $\mathbf{9 5 \%} \mathbf{C l}$ \\
\hline Amisulpride & 8 & 1.24 & $\mathbf{1 . 1 2 - 1 . 9 4}$ & -6.28 & $-136.12-35.69$ \\
Aripiprazole & 14 & 0.99 & $0.81-1.22$ & 3.83 & $-27.32-24.64$ \\
Clozapine & 11 & 1.01 & $0.92-1.13$ & 5.19 & $-13.96-27.07$ \\
Norclozapine & 12 & 0.94 & $0.90-1.04$ & 6.22 & $-6.87-13.10$ \\
Quetiapine & 26 & 1.09 & $\mathbf{1 . 0 2 - 1 . 1 4}$ & -2.99 & $-\mathbf{6 . 0 2 - - 1 . 1 5}$ \\
7OH-N-desalkylquetiapine & 25 & 1.44 & $\mathbf{1 . 0 6 - 2 . 9 8}$ & -2.59 & $-\mathbf{9 . 7 9 - - 0 . 4 8}$ \\
7OH-quetiapine & 23 & 0.80 & $0.65-1.08$ & 0.38 & $-0.54-1.51$ \\
Paliperidone & 36 & 1.12 & $\mathbf{1 . 0 6 - 1 . 2 0}$ & 0.17 & $-1.25-1.11$ \\
Risperidone & 21 & 1.06 & $0.96-1.12$ & 0.20 & $-0.21-0.91$
\end{tabular}

Table 6: Result of Passing Bablok regression analysis for the comparison between v-DBS and c-DBS. The $95 \%$ confidence intervals $(\mathrm{Cl})$ of the slope that do not contain 1 and $95 \% \mathrm{Cl}$ of the intercept that did not contain 0 are marked in bold.

\begin{tabular}{lccccc} 
Analyte & No of samples & Slope & $\mathbf{9 5 \%} \mathbf{C l}$ & Intercept & $\mathbf{9 5 \% ~ C l}$ \\
\hline Amisulpride & 8 & 1.05 & $0.89-1.65$ & 8.99 & $-99.66-62.88$ \\
Aripiprazole & 14 & 0.99 & $0.91-1.07$ & 4.37 & $-6.51-15.56$ \\
Clozapine & 11 & 1.03 & $0.94-1.11$ & -1.00 & $-11.75-17.26$ \\
Norclozapine & 12 & 1.05 & $0.97-1.11$ & 1.24 & $-9.04-10.69$ \\
Paliperidone & 36 & 1.06 & $1.01-1.13$ & 0.46 & $-0.48-1.35$ \\
Quetiapine & 26 & 0.94 & $0.91-0.99$ & 0.65 & $-0.67-1.91$ \\
7OH-N-desalkylquetiapine & 23 & 0.90 & $0.82-1.04$ & 0.20 & $-0.74-0.60$ \\
7OH-quetiapine & 22 & 0.97 & $0.85-1.05$ & -0.16 & $-0.72-0.27$ \\
Risperidone & 13 & 1.02 & $0.99-1.11$ & 0.31 & $-0.59-0.68$
\end{tabular}

Table 7: Comparison of the clinical interpretation of the obtained blood levels (based on the therapeutic ranges defined by TIAFT and the AGNP-TDM guidelines) from serum and venous whole blood analyzed by the conventional method and venous and capillary blood analyzed by the DBS- 
method ( $v$-DBS and c-DBS). Data from reduced haloperidol and $7 \mathrm{OH}-\mathrm{N}$-desalkylquetiapine were not included, since no therapeutic ranges are available.

\begin{tabular}{lrrrr} 
& \multicolumn{1}{c}{ Blood vs. v-DBS } & Blood vs. c-DBS & v-DBS vs. c-DBS & Serum vs. c-DBS \\
\hline Identical interpretation & 214 & 159 & 162 & 152 \\
< therapeutic range & 11 & 6 & 10 & 8 \\
within therapeutic range & 49 & 38 & 37 & 33 \\
$\quad>$ therapeutic range & 139 & 105 & 103 & 103 \\
Different interpretation & 15 & 10 & 12 & 8 \\
Total & 18 & 7 & 4 & 14 \\
\hline Sensitivity (\%) & 232 & 166 & 166 & 166 \\
\hline
\end{tabular}

